\title{
Surface Reactivity of Carbonaceous Nanoparticles: The Importance of Surface Pocket
}

\author{
Hongyu Wang, Xiaoya Chang and Dongping Chen * \\ State Key Lab of Explosion Science and Technology, Beijing Institute of Technology, Beijing, China
}

\section{OPEN ACCESS}

Edited by:

Hongsheng Guo,

National Research Council Canada

(NRC-CNRC), Canada

Reviewed by:

Jianfei $X i$,

Nanjing Normal University, China Nickolas Eaves,

University of Windsor, Canada

*Correspondence:

Dongping Chen

dc516@bit.edu.cn

Specialty section:

This article was submitted to Engine and Automotive Engineering, a section of the journal

Frontiers in Mechanical Engineering

Received: 08 July 2021 Accepted: 05 August 2021

Published: 27 August 2021

Citation:

Wang H, Chang X and Chen D (2021) Surface Reactivity of Carbonaceous Nanoparticles: The Importance of

Surface Pocket.

Front. Mech. Eng 7:738354. doi: 10.3389/fmech.2021.738354
The surface reactivity of carbonaceous nanoparticles is revealed from the barrier height and reaction enthalpy of hydrogen abstraction reaction by $\mathrm{H}$ radicals computed at the M06-2X/6-311g(d,p)//B3LYP/6-311G(d,p) level of theory. Small polycyclic aromatic hydrocarbon (PAH) clusters are selected as the model system of carbonaceous nanoparticles. The PAHs considered are naphthalene, pyrene, coronene, ovalene and circumcoronene. Cluster sizes range from dimer to tetramer with a parallel or crossed configuration. All results show similar values as that of monomers, but naphthalene dimers with a crossed configuration yield a lower barrier height and reaction enthalpy by $\sim 2 \mathrm{kcal} /$ mol. A minor size dependence is noticed in the series of naphthalene clusters where a larger cluster exhibits a smaller barrier height. Larger homogeneous PAH clusters in a size range of $1.1-1.9 \mathrm{~nm}$ are later generated to mimic nascent soot surface. It is found that the barrier height decreases with the increase in particle size, and the averaged values are $\sim 2 \mathrm{kcal} / \mathrm{mol}$ lower than that of monomers. More importantly, a larger particle shows a wider spread in barrier heights, and low barrier heights are seen in the surface shallow regions (e.g., surface pockets). The lowest barrier height of $\sim 8.5 \mathrm{kcal} / \mathrm{mol}$ is observed at a $\mathrm{C}-\mathrm{H}$ site locating in a surface pocket. A set of model systems are built to reveal the underlying mechanism of reduction in barrier height. It is shown that the reduction is caused by local interactions between the neighboring atoms and the local curvature. Further analysis on the average localized ionization potential shows that larger particles have higher reactivity, further supporting our findings from the barrier height of hydrogen abstraction reactions. Therefore, it is concluded that the surface reactivity depends on the particle size and the most reactive sites always locate at the surface pockets.

Keywords: carbonaceous nanoparticles, DFT, energy barriers, $\mathrm{H}$ abstraction, surface reactions

\section{INTRODUCTION}

Surface reaction is a key process in the mass growth of soot formation (Frenklach and Wang, 1991; Frenklach, 1996; Appel et al., 2000; Wang et al., 2015). For instance, the predicted particle size distributions in a stretch-stabilized ethylene flames are ultra-sensitive to the parameters in surface reactions (Camacho et al., 2017). Besides mass growth, surface reactions also account for soot oxidation (Camacho et al., 2015), which relates to the mitigation of soot emission in practical combustors. The oxidative reactivity of soot particles formed from conventional and reformulated fuels has complex impacts on the fuel consumption, engine performance, lubricant oil degradation in the road vehicles (Sharma et al., 2016). Recently, Lapuerta and the coworkers (Lapuerta et al., 2020) 
reviewed the soot reactivity on diesel filter regeneration, suggesting that surface properties, i.e., surface area, solid-gas reactions and soot porosity, determine the regeneration process.

In soot models, surface reactions were usually treated as the analogy of gas-phase reactions, i.e., a reaction of polycyclic aromatic hydrocarbon (PAH) and radicals. However, not all soot surface can react with radicals at flame conditions, a steric parameter $\alpha$ was therefore proposed by Frenklach and Wang (1991) to account for the probability of successful collisions on the reactive sites. In the following works, parameter $\alpha$ was refitted against a wide range of experimental results, such as soot volume fraction (Frenklach and Wang, 1991; Dworkin et al., 2011; Khosousi and Dworkin, 2015), but none of the works agree with each other. Recently, we extracted the parameter $\alpha$ from microscopic representations of nascent soot using molecular dynamics method (Chen and Luo, 2020), and our work demonstrated that large uncertainties exist in the parameters of surface reactions, such as the steric parameter $\alpha$ and the active number density of active sites $\chi_{\mathrm{i}}$. The impact of the uncertainties on reaction rates are equivalent to the reduction of the energy barrier of surface reactions by $7.2 \mathrm{kcal} / \mathrm{mol}$ at $2000 \mathrm{~K}$. It is worth noting that the barrier heights in surface reactions is never directly estimated and it is always assumed to be the same as the analogous gas-phase reaction in the last three decades.

The key pathway of mass growth in surface reaction is governed by the well-known HACA mechanism (Appel et al., 2000), where the Arrhenius parameters of hydrogen abstraction and $\mathrm{C}_{2} \mathrm{H}_{2}$ addition reactions are directly taken from the gas-phase reaction. The barrier heights $\left(\Delta E_{0}\right)$ and reaction enthalpies $\left(\Delta H_{0}\right)$ of hydrogen abstraction from planar $\mathrm{PAHs}$ with $\mathrm{H}$ radicals (Hou and You, 2017) are well benchmarked using the CCSD(T)/CBS level of theory. In particular, the $\Delta E_{0}$ and $\Delta H_{0}$ for benzene is 15.6 and $7.9 \mathrm{kcal} / \mathrm{mol}$, respectively. Also, hydrogen abstraction reactions on aromatics exhibit a minor site dependence (Liu $\mathrm{P}$. et al., 2019). In the formulation of ABF model (Appel et al., 2000), the reactive $\mathrm{C}-\mathrm{H}$ site on particle surface locates at the aromatic bay. From density functional theory (DFT) calculations, C-H bonds locating at the aromatic bay exhibit a repulsion from the neighboring atoms, and the bond dissociation energy is typically reduced by $1-3 \mathrm{kcal} / \mathrm{mol}$ (Cioslowski et al., 1996). Therefore, Appel et al. (2000) reduced the activation energy of $\mathrm{H}$ abstraction reaction on particle surface by $3 \mathrm{kcal} / \mathrm{mol}$ to account for the above effect.

The potential energy surface (PES) of soot particle significantly differs from PAH molecules, which should impact the surface reactivity. The soot TEM images (Wan et al., 2018; Pfau et al., 2020) showed that the surface is made of various defects. In the catalysis community, it is well known that the crystal phase impacts the catalytic activity. Liu et al. (2017) reviewed the effects of crystal phase in Fischer-Tropsch catalysts, for example, cobalt, ruthenium, iron, iron carbides and nickel nanoparticles; the catalytic capacities show a clear dependence on the crystal phase. Similarly, in the lowtemperature CO oxidation ( $\mathrm{Li}$ et al., 2014), the catalytic activities of anatase is much higher than that of rutile in the tested conditions. Also, surface defects (e.g., kinks and steps) on the catalyst surface could provide active sites for surface reactions to enhance reactivity and even selectivity (Chen et al., 2019; Kopač et al., 2019). Although soot is a type of amorphous material and does not show a well-defined crystal structure, the knowledge derived from catalysis community makes us to question whether complex surface (local textural arrangements) of nascent soot impacts the reaction kinetics. This issue would justify the reduction in the surface reactions in the $\mathrm{ABF}$ model. Besides, surface reactions for mass growth always occur on the boundary of particles (Grančič et al., 2016) due to the geometric effect. By contrast, soot particles might undergo internal oxidation reactions under oxygen-rich environments (Sediako et al., 2017; Macián et al., 2019), and the complex potential energy surface inside a particle must impact the energy barrier to an extent. Nevertheless, the energy barrier of surface reaction should be lower than that occurring in the gas-phase reaction due to interactions from the neighboring components.

The development of soot molecular representations started from the homogeneous clusters of planar PAHs up to $\sim 8 \mathrm{~nm}$ in diameter (Chen et al., 2014; Chen et al., 2015b). Bowal et al. (2019a) extended the previous attempt and successfully created heterogenous clusters of planar PAHs, for example, a cluster with 50 circumcoronene and 50 coronene. Later, clusters with curved PAHs, i.e., corannulene, were generated from molecular dynamic simulations (Bowal et al., 2019b). Recently, heterogeneous clusters with cross-linked PAHs (Pascazio et al., 2019) were available using reactive molecular dynamic simulations. The applications of these model systems of soot can be seen in the solid-liquid morphology transition (Chen et al., 2014; Chen et al., 2015b), surface reactivity (Chen and Luo, 2020) and also optical properties (Liu C. et al., 2019; Chen and Wang, 2019b). In this work, we explore the energy barriers of hydrogen abstraction reactions from a set of model systems of soot by $\mathrm{H}$ radicals. The clusters with planar PAHs are selected as the model system to mimic the complex soot surface. The DFT method is used to compute the energy barriers of small $\mathrm{PAH}$ clusters with five PAHs, i.e., naphthalene, pyrene, coronene, ovalene and circumcoronene. A few PAH clusters with up to 15 monomers are generated to represent nascent soot, and their energy barriers for hydrogen abstraction reactions are estimated using a frozen algorithm. The size dependence of energy barriers is then discussed, and the location with reduced barrier heights are further examined in detail. Also, a set of model systems are built to reveal the physics behind the reductions in barrier heights. Last but not the least, the analysis of average local ionization potential is carried out to support our findings.

\section{COMPUTATIONAL METHODS}

The dimer, trimer and tetramer of naphthalene and pyrene together with the dimers of coronene are generated from geometry optimizations at the B3LYP/6-311G(d,p) level of theory with an empirical dispersion correction (Grimme et al., 2010). The dimers of ovalene and circumcoronene are optimized at the $\mathrm{B} 3 \mathrm{LYP} / 6-31 \mathrm{G}(\mathrm{d})$ level of theory to reduce the computational cost. All optimized geometries are confirmed by analyzing vibrational frequencies, and the ZPE vibrational energy 
A Parallel configurations:

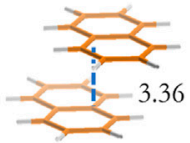

Napthalene $_{2}$

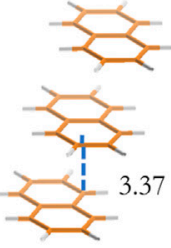

Napthalene $_{3}$

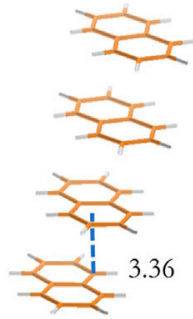

Napthalene $_{4}$

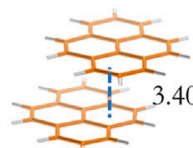

Pyrene $_{2}$

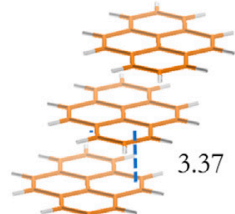

Pyrene $_{3}$

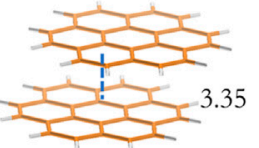

Coronene $_{2}$

\section{B Crossed configurations:}

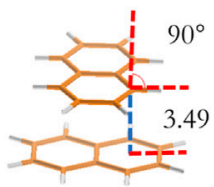

Napthalene $_{2}$

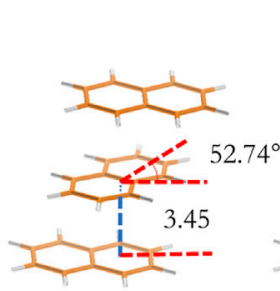

Napthalene $_{3}$

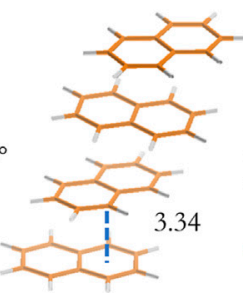

Napthalene $_{4}$

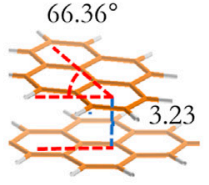

Pyrene $_{2}$

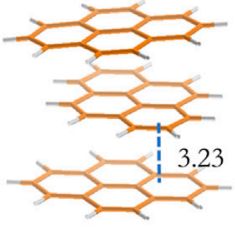

Pyrene $_{3}$

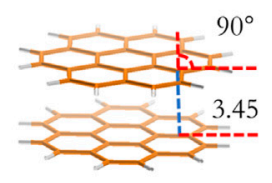

Coronene $_{2}$

FIGURE 1 | Optimized configurations of naphthalene clusters. The size of cluster from left to right is dimer to tetramer. The upper and lower panel presents a local minimum with a parallel $(\mathbf{A})$ and a crossed $\mathbf{( B )}$ configuration, respectively. The layer separation and the rotation angle between planes are indicated on the figure.

is scaled by 0.977 (Andersson and Uvdal, 2005). The optimized configurations of naphthalene, pyrene and coronene clusters from dimer to tetramer are shown in Figure 1, and configurations of other $\mathrm{PAH}$ clusters are included in the supplemental material (Supplementary Figure S1). The layer separation lies in the range of $3.3 \AA$ and $3.5 \AA$ depending on the size of monomers.

Considering the multiple sites of surface reactions, an in-house python code is applied to generate initial configurations for both transition state and product optimization at each potential site for hydrogen abstraction reactions automatically. In particular, surface atoms are identified using the solvent-excluded surface with a probe size as $2.0 \AA$ (Chen et al., 2015a; Chen and Luo, 2020). The barrier heights and reaction enthalpies (0 K) for hydrogen abstraction reactions are derived from single-point energy calculations at the M06-2X/6-311G(d,p) level of theory with an empirical dispersion correction (Grimme et al., 2010). The accuracy of our method was evaluated in a previous work (Hou and You, 2017), and the uncertainties compared to the CCSD(T)/CBS level of theory were less than $1 \mathrm{kcal} / \mathrm{mol}$ for the barrier heights and reaction enthalpies $(0 \mathrm{~K})$ of $\mathrm{H}$ abstraction reaction on the benzene and naphthalene. Gaussian 09 program is used to carry out the DFT calculations (Frisch et al., 2009).

The multi-configurational character of $\mathrm{PAH}$ clusters is investigated by considering $\mathrm{T} 1$ diagnostics at the $\operatorname{CCSD}(\mathrm{T}) / \mathrm{cc}$ pVDZ level of theory. In particular, a naphthalene trimer with a parallel configuration is selected to react with $\mathrm{H}$ radical. All of the T1 diagnostics for the reactant, the product and the transition state are less than 0.02, which supports our application of the single-reference method for single-point energy calculations ( $\mathrm{Yu}$ and Truhlar, 2014).

Besides the optimized structures, we generate six larger $\mathrm{PAH}$ clusters to mimic the complex surface of carbonaceous nanoparticles, including naphthalene ${ }_{10}$, naphthalene ${ }_{15}$, pyrene $_{5}$, pyrene $_{10}$, coroene ${ }_{5}$, and coronene $e_{10}$ clusters. The corresponding particle diameter is $1.47,1.71,1.31,1.69,1.46$, and $1.87 \mathrm{~nm}$, respectively. These clusters are equilibrated using $\mathrm{MD}$ simulations above the melting points of each configuration (Chen et al., 2014; Chen et al., 2015b), and the selected temperatures lie in the range of $500-1000 \mathrm{~K}$. The potential functions for $\mathrm{C}-\mathrm{C}, \mathrm{C}-\mathrm{H}$ and $\mathrm{H}-\mathrm{H}$ interactions are described by the isoPAHAP potential (Totton et al., 2012). Note the configurations taken from $\mathrm{MD}$ trajectories correspond to a transient structure rather than a locally preferred structure. The morphology of these clusters (Figure 2) is consistent with the liquid-like structures in previous works (Chen et al., 2014; Chen et al., 2015b). An additional configuration (e.g., naphthalene ${ }_{15,2}$ ) is also included, and its results agree with those of naphthalene ${ }_{15}$ (see Supplementary Figure S2).

The saddle point for hydrogen abstraction reactions on the models of nascent soot (Figure 2) is investigated by considering only the $\mathrm{H}$ radical and the substituted hydrogen atom during the optimization process, while other atoms are frozen to reduce the computational costs by at least one order of magnitude. The computational error of the current frozen scheme is examined using a naphthalene tetramer, a pyrene trimer and a coronene 


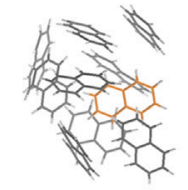

Naphthalene $_{10}$

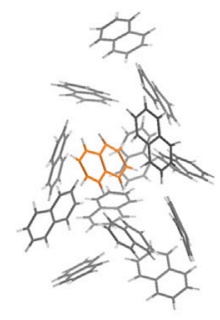

Naphthalene $_{15}$

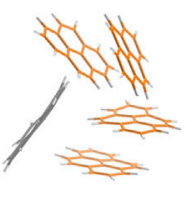

Pyrene $_{5}$

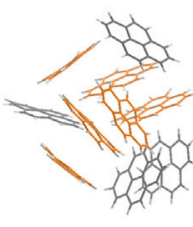

Pyrene $_{10}$

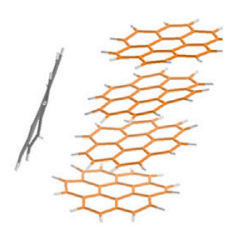

Coronene $_{5}$

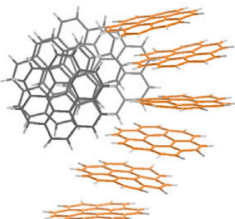

Coronene $_{10}$

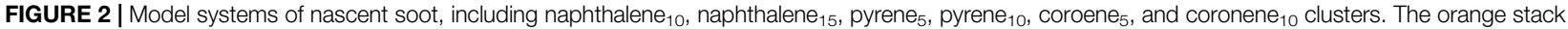
highlights the maximum stacks in the configurations.

TABLE 1 | The errors in energy barriers of transition states using different frozen schemes and the respective ZPE corrections. ${ }^{a}$

\begin{tabular}{|c|c|c|c|}
\hline Case & $\begin{array}{l}\text { Errors in the } \\
\text { selected frozen scheme }\end{array}$ & $\begin{array}{c}\text { Errors in the frozen scheme with } \\
3 \AA \text { as the criteria }\end{array}$ & ZPE corrections \\
\hline napthalene $_{4}{ }^{d}$ & $2.56 \pm 0.13$ & $1.83 \pm 0.12$ & $1.73 \pm 0.08$ \\
\hline pyrene $_{3}{ }^{d}$ & $2.55 \pm 0.16$ & $1.85 \pm 0.14$ & $1.77 \pm 0.10$ \\
\hline coronene $_{2}^{d}$ & $2.40 \pm 0.28$ & - & $1.76 \pm 0.08$ \\
\hline
\end{tabular}

${ }^{a}$ All energies are in $\mathrm{kcal} / \mathrm{mol}$. Only the parallel configurations are considered. The uncertainties refer to one standard deviation in the predictions of energy barriers.

${ }^{b}$ Only the hydrogen radical and the abstracted hydrogen atom are not frozen in the searching of the saddle point.

${ }^{c}$ The hydrogen radical and any neighboring atoms within $3 \AA$ away from the radical are not frozen in the searching of the saddle point.

${ }^{d} 32,30$, and 24 transition states are explored for napthalene 4 , pyrene ${ }_{3}$ and coronene ${ }_{2}$ clusters, respectively. The \pm values refer to one standard deviation in the predicted errors.
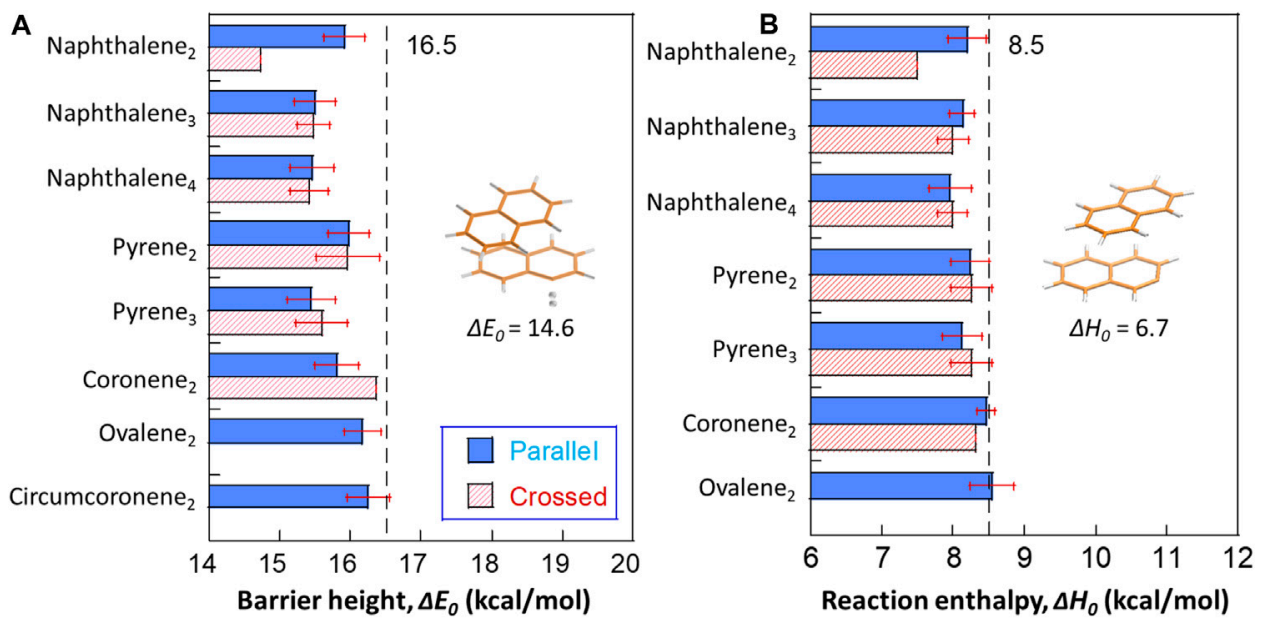

FIGURE 3 | The barrier heights (A) and reaction energies (B) for hydrogen abstraction from small PAH clusters by H radicals. The transition state of a naphthalene dimer with the lowest barrier height among all optimized configurations is highlighted in Figure $\mathbf{3 A}$. The structure showing the least reaction enthalpy for $\mathrm{H}$ abstraction reaction is included in Figure 3B. The dashed lines refer to the average barrier heights and reaction energies of optimized monomers at the M06-2X/6-311G(d,p) level of theory as 16.5 and $8.5 \mathrm{kcal} / \mathrm{mol}$, respectively. The red error bars refer to one standard deviation from the results on different sites within the particular case.

dimer with a parallel configuration (Table $\mathbf{1}$ ). The full optimized transition state calculations at the M06-2X/6-311G(d,p) level of theory are treated as the benchmark to evaluate the uncertainty in our frozen algorithm. The selected frozen scheme overestimates the barrier height by $2.50 \mathrm{kcal} / \mathrm{mol}$ on average, which is mainly due to the zero-point energy (ZPE) correction $(1.75 \mathrm{kcal} / \mathrm{mol})$. Although the errors in the frozen scheme can be reduced to the level of ZPE corrections by applying a distance threshold of 3 Å to include more atoms in the optimization, it is still not recommended because the searching algorithm of transition states would pull back the neighbouring hydrogen atoms to the equilibrated position of $\mathrm{C}-\mathrm{H}$ bonds and significantly reduce the overall barrier heights. Note that the clusters in this work are snapshots sampled at high temperatures, and the neighbouring hydrogen atoms in $\mathrm{C}-\mathrm{H}$ bonds might sit far away from the equilibrated position. It is evident that the 

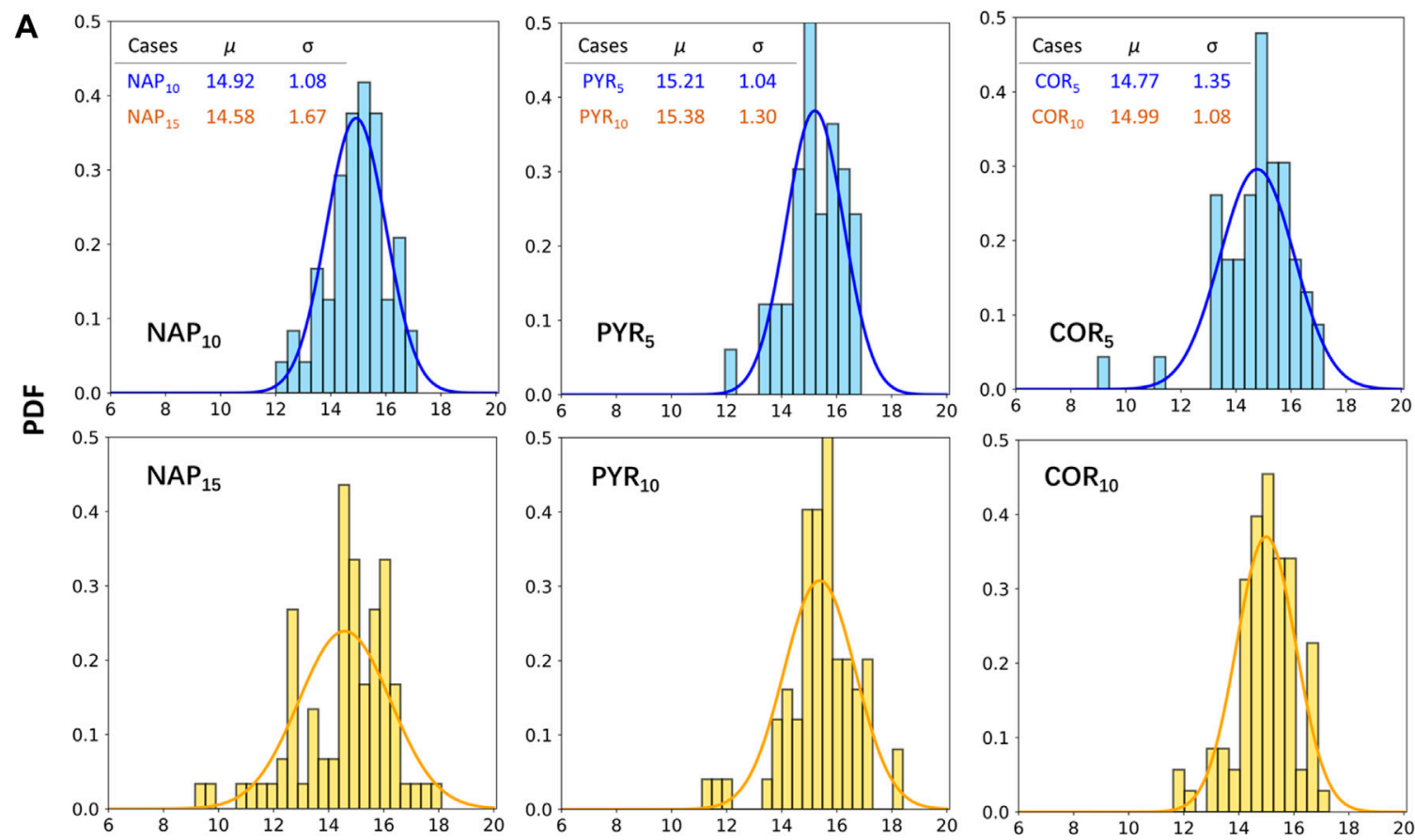

B

Barrier height, $\Delta E_{0}(\mathrm{kcal} / \mathrm{mol})$
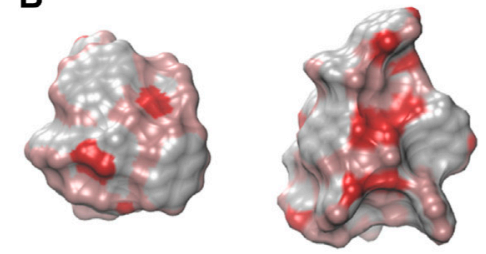

$\mathrm{NAP}_{10}$

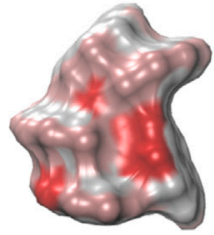

$\mathrm{PYR}_{5}$

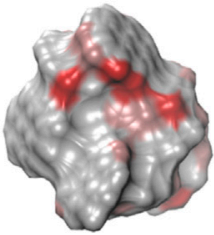

$\mathrm{PYR}_{10}$
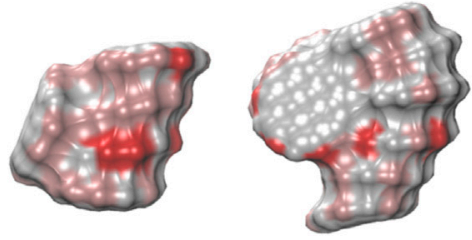

$\mathrm{COR}_{5}$

$\mathrm{COR}_{10}$

Light red: $\Delta E_{0}>13.5 \mathrm{kcal} / \mathrm{mol}$

Dark red: $\Delta E_{0}<13.5 \mathrm{kcal} / \mathrm{mol}$

FIGURE 4 | (A) The barrier heights for hydrogen abstractions from selected naphthalene (NAP), pyrene (PYR) and coronene (COR) clusters by H radicals. The blue bars represent the results of the smaller clusters, while the larger ones are illustrated by yellow bars. A correction of $2.5 \mathrm{kcal} / \mathrm{mol}$ is included to account for the errors in the frozen algorithm. $\mu$ and $\sigma$ represent the mean and standard deviation of the fitted gaussian distributions. (B) The spatial distributions of barrier heights on each surface hydrogen atoms. The white, light red and dark red refer to surface carbons, surface hydrogens with $\Delta E_{O}>13.5 \mathrm{kcal} / \mathrm{mol}$, and surface hydrogens with $\Delta E_{O}<13.5 \mathrm{kcal} / \mathrm{mol}$, respectively.

frozen scheme with $3 \AA$ as the critical criteria yields unphysical barrier heights as $3.5 \mathrm{kcal} / \mathrm{mol}$ for the napthalene ${ }_{15}$ cluster (Supplementary Figure S3).

\section{RESULTS AND DISCUSSION}

We report DFT calculations of barrier heights and reaction enthalpies $(0 \mathrm{~K})$ for hydrogen abstraction reactions at all potential sites of small naphthalene, pyrene, coronene, ovalene and circumcoronene clusters as shown in Figure 3. The results show that the calculated $\Delta E_{0}$ are very close to that of monomers (e.g., $16.5 \mathrm{kcal} / \mathrm{mol}$ of benzene), and the dimer data falls in the similar range as a previous work (Hou and You, 2017). A naphthalene dimer with a crossed configuration yields a smaller value as $14.6 \mathrm{kcal} / \mathrm{mol}$, while the $\Delta E_{0}$ of its counterpart with a parallel configuration is $15.9 \mathrm{kcal} / \mathrm{mol}$. By contrast, the crossed configuration of coronene dimers have a higher $\Delta E_{0}$ than the parallel configuration. Other cases show no differences considering both isomers. The obvious difference between reaction barriers of crossed and parallel naphthalene dimers can be attributed the significant geometry change in the transition state (see the configurations in Figure 3). For a naphthalene dimer with a crossed configuration, it rotates along the central plane by $\sim 30^{\circ}$ to access the transition state. However, no such rotation is seen in the trimer and tetramer. One interesting trend should be emphasized here; $\Delta E_{0}$ is weakly dependent on the particle size. As the size grows from dimer to tetramer of naphthalene, a reduced 


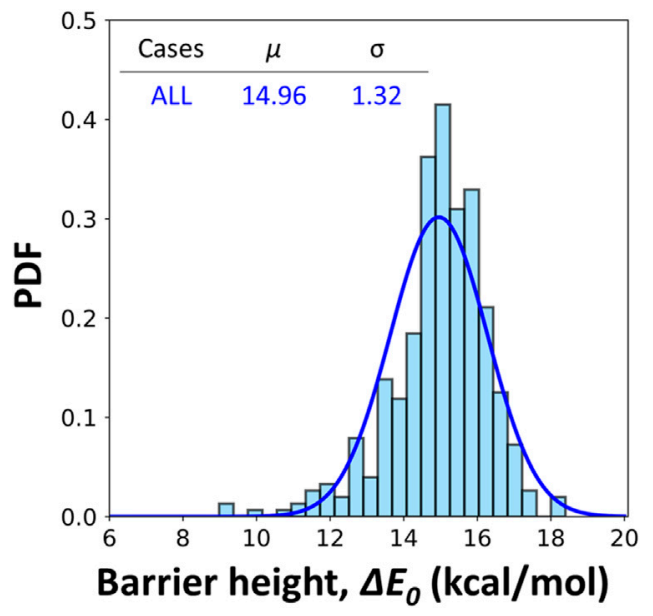

FIGURE 5 | Fitted gaussian distribution of barrier heights for hydrogen abstractions by $\mathrm{H}$ radicals including $\mathrm{NAP}_{10}, \mathrm{NAP}_{15}, \mathrm{PYR}_{5}, \mathrm{PYR}_{10}, \mathrm{COR}_{5}$ and $\mathrm{COR}_{10}$ clusters. A correction of $2.5 \mathrm{kcal} / \mathrm{mol}$ is included to account for the errors in the frozen algorithm. $\mu$ and $\sigma$ represents the mean and standard deviation of the fitted distributions.

$\Delta E_{0}$ is observed, and pyrene cases follow this trend. Although the reduction is minor $(\sim 1 \mathrm{kcal} / \mathrm{mol})$, this raises a question whether this dependence can be extrapolated to larger sizes.

For reaction enthalpies $(0 \mathrm{~K})$, most of the computed values are close to the values of monomers (e.g., $8.5 \mathrm{kcal} / \mathrm{mol}$ ). Again, naphthalene dimers with a crossed configuration exhibit the lowest reaction enthalpy as $6.7 \mathrm{kcal} / \mathrm{mol}$. It was found in previous works (Cioslowski et al., 1996; Hou and You, 2017) that the hydrogens locating at the bay site exhibit repulsive forces weakening the $\mathrm{C}-\mathrm{H}$ bonds by $1-3 \mathrm{kcal} / \mathrm{mol}$. However, the reduction in naphthalene dimers with a crossed configuration does not share the same physical reason. In our case, the layer separation is $3.49 \AA$ (Figure 1), falling in the weak attraction region, and thus the neighboring hydrogens do not experience repulsions. Instead, an obvious intermolecular deformation is seen in the product of abstraction reaction, and this preferred complex yields a reduced energy resulting the reduction in the overall $\Delta H_{0}$. Also, no size dependence is observed in reaction enthalpies.

Six larger PAH clusters with sizes from 1.3 to $1.9 \mathrm{~nm}$ in diameter are further selected to mimic the surface of nascent soot (Figure 2). The reported energy barriers are deducted by $2.5 \mathrm{kcal} / \mathrm{mol}$ as a correction taken from the frozen algorithm. The average $\Delta E_{0}$ of all six cases fall in the range of $14.4-15.1 \mathrm{kcal} / \mathrm{mol}$, and again, it is evident that the $\Delta E_{0}$ of the abstraction reaction shows a minor size dependence. A larger particle has a wider spread in the $\Delta E_{0}$. For example, the sigma value of the $\mathrm{NAP}_{15}$ cluster is roughly doubled compared to the smaller counterpart. The magnitude of spread correlates with surface complexity, and a larger surface usually exhibits more textual arrangements. It is found that only $\sim 10 \%$ of all calculated cases have a $\Delta E_{0}$ higher than $16.5 \mathrm{kcal} / \mathrm{mol}$, suggesting that the surface reactivity of soot always higher than that of monomers. Also highlighted in

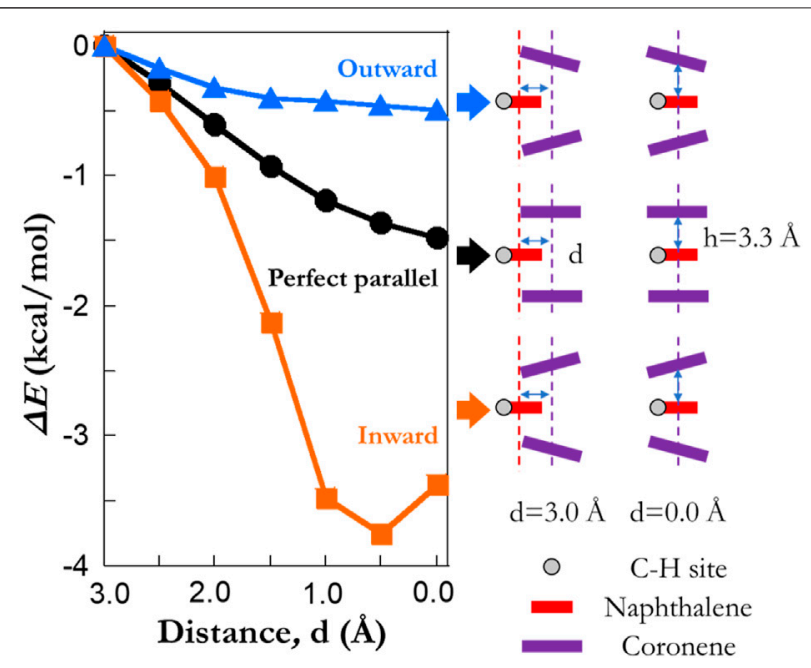

FIGURE 6 | The impact of local textural arrangements on the barrier heights of hydrogen abstraction reactions by a $\mathrm{H}$ radical. A model system with two coronene molecules and one naphthalene is considered. The blue, black and orange lines refer to the results of a "outward", a "perfect parallel" and an "inward" configuration, respectively. The rotation angle for both "outward" and "inward" cases is 15 degree. "C-H site" highlights the abstracted hydrogen atoms from model systems. Note that the results are extracted using the frozen scheme.

Figure $4 \mathrm{~B}$ is the spatial distribution of $\Delta E_{0}$ on each cluster. We classify the data into two cases for discussion, e.g., $\Delta E_{0}>13.5 \mathrm{kcal} / \mathrm{mol}$ and $\Delta E_{0}<13.5 \mathrm{kcal} / \mathrm{mol}$. It is shown that most of the edge hydrogens on stacks fall in the former cases, and their reactivity is close to the monomer. At some surface shallow regions (e.g., pockets (Chen et al., 2015a)), the $\Delta E_{0}$ is reduced by at least $3 \mathrm{kcal} / \mathrm{mol}$, which cannot be explained by the molecular repulsion in monomers (Cioslowski et al., 1996; Hou and You, 2017). In the original ABF model (Appel et al., 2000), a reduction of $3 \mathrm{kcal} / \mathrm{mol}$ is applied to account for the effect of molecular repulsion. Our results show that the $\Delta E_{0}$ of abstraction reactions at the surface pockets would require further reduction. For example, the lowest $\Delta E_{0}$ seen in all cases is $\sim 9.0 \mathrm{kcal} / \mathrm{mol}$ (about the half of the barrier seen in monomers). Together with the weak dependence on the particle size, we would expect a lower $\Delta E_{0}$ if extrapolating the particle size to larger values. However, the selected clusters in this work are smaller than $2 \mathrm{~nm}$ in diameter. It is still questionable whether the weak dependence of surface reactivity on the particle size can be extracted to larger soot particles. This size dependence of surface reactivity still requires future investigations.

Although a Gaussian distribution is fitted in Figure 4, we do not argue that the underlying distribution must be Gaussianlike. Instead, obvious failure can be noticed from both the cases of pyrene and coronene clusters. The fitted distributions here serve as the guidance to quantitively compare the different cases. Figure $\mathbf{5}$ includes all data from the six simplified soot models. The reported barrier height is $14.96 \pm 1.32 \mathrm{kcal} / \mathrm{mol}$. In all data, the number of cases with a barrier height lower than $13.5 \mathrm{kcal} / \mathrm{mol}$ is $12.15 \%$. The detailed distributions of $\Delta E_{0}$ for each case are included in 


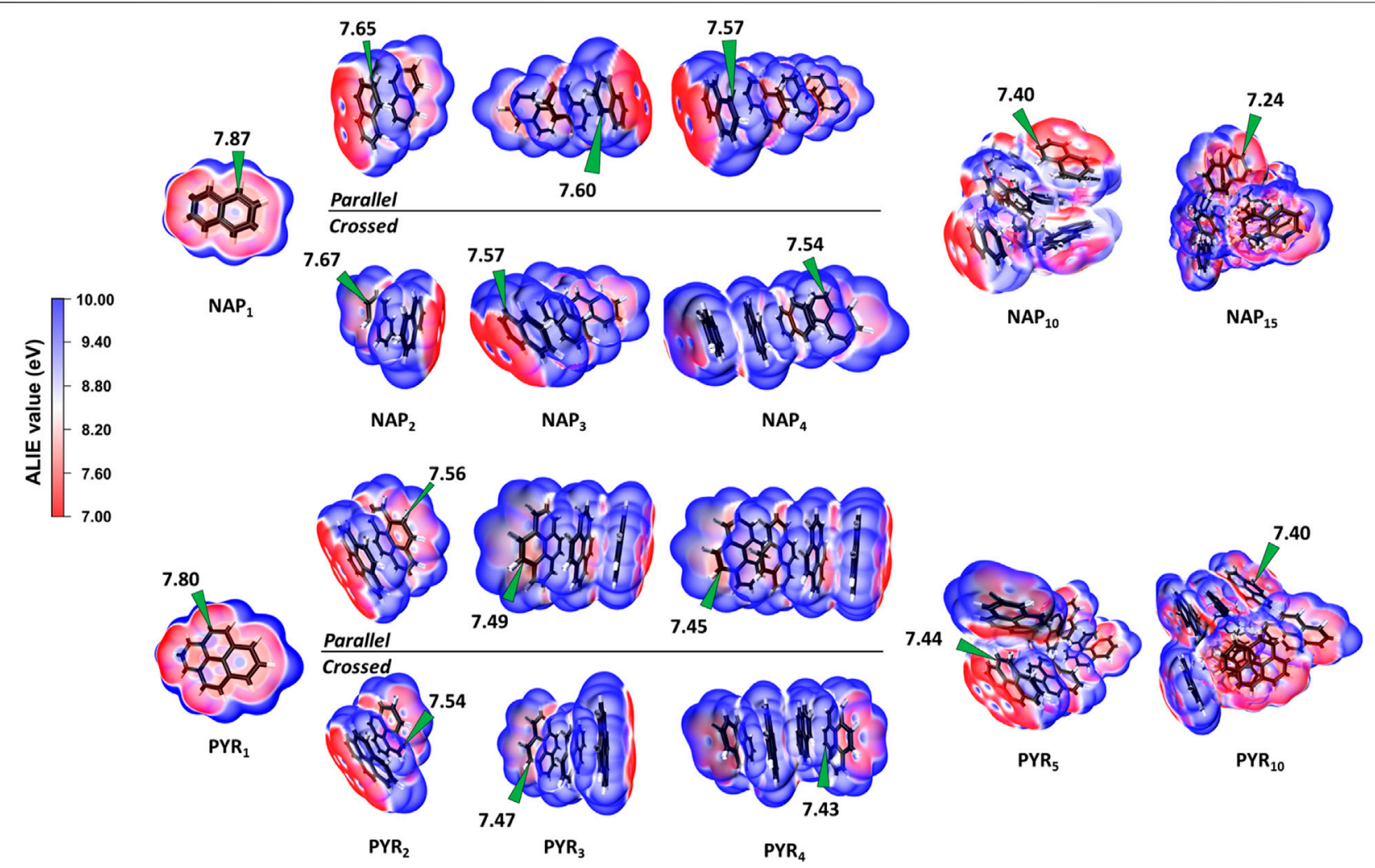

FIGURE 7 | Local ionization energy of NAP and PYR clusters from monomer up to 15 components calculated at the B97D/6-311G(d,p) level of theory. The minimums of ALIE values are highlighted on the plot. The both parallel and crossed configurations are considered for dimers, trimers and tetramers.

Supplementary Figure S4. Most of the cases are well covered by the treatment in original ABF model (Appel et al., 2000), however, the importance of these low-value barrier height remains uncertain for future reference.

We then take a step further to investigate the reduction in $\Delta E_{0}$. Model systems of $\mathrm{COR}_{2} \mathrm{NAP}_{1}$ clusters are generated with three different arrangements (Figure 6): 1) "perfect parallel", 2) a stack of three PAHs with the angle between the upper coronene and naphthalene as $15^{\circ}$ (termed as the "outward"), 3) a stack of three PAHs with the angle between the upper coronene and naphthalene as $15^{\circ}$ (termed as the "inward"). In all cases, the layer separation between planes is kept as $3.3 \AA$ and the lower coronene is the mirror image of the upper one. The frozen algorithm with a distance threshold of $3 \AA$ is again used to calculate the barrier heights of $\mathrm{COR}_{2} \mathrm{NAP}_{1}$ clusters. It is found that the reduction of barrier height depends on the relative distance between the naphthalene and the other coronene molecules. A reduction in the barrier height is observed when moving naphthalene inward in all three cases. The local curvature is also an important factor; the "inward" cases yield a reduction of $\sim 4 \mathrm{kcal} / \mathrm{mol}$, while the maximum reduction in the "outward" is $\sim 0.5 \mathrm{kcal} / \mathrm{mol}$. In the "inward" cases, the distance between atoms in coronene and the abstracted site could be as low as $2.6 \AA$, which is much smaller than that of other cases. At such conditions, the abstracted site exhibits a strong repulsive force from neighboring species, and this corresponds to the bay site (Cioslowski et al., 1996; Hou and You, 2017) causing a reduction in barrier height. However, both "perfect parallel" and "outward" cases lie in the attraction regions, and the repulsion mechanism cannot explain the moderate reductions in barrier height. This phenomenon may relate to the delocalization of $p i$ electrons which weakens the $\mathrm{C}-\mathrm{H}$ bonds or the formation of weak hydrogen bonds $(<4 \mathrm{kcal} /$ $\mathrm{mol}$ ). Although this set of model systems does not yield a large reduction as $\sim 8 \mathrm{kcal} / \mathrm{mol}$, it reveals the fundamental reason in the reductions of barrier height. Considering larger particles with more complex surface, we expect to see a wide range of possible configurations, which might be the analogies of the simplified cases.

Average local ionization energy (ALIE) (Sjoberg et al., 1990) describes the site reactivity of electrophilic or radical attack on aromatic species. Here, we include the ALIE analysis to examine the surface reactivity of our model systems. The ALIE is computed as

$$
\bar{I}(\boldsymbol{r})=\sum_{i} \frac{\rho_{i}(\boldsymbol{r})\left|\varepsilon_{i}\right|}{\rho_{\mathrm{tot}}(\boldsymbol{r})}
$$

where $\rho_{i}(\boldsymbol{r})$ is the electronic density of the $i$ th molecular orbital at the point $\boldsymbol{r}, \rho_{\text {tot }}(\boldsymbol{r})$ is the total electronic density at the point $\boldsymbol{r}$ and $\left|\varepsilon_{i}\right|$ is the orbital energy. The electron density of NAP and PYR 


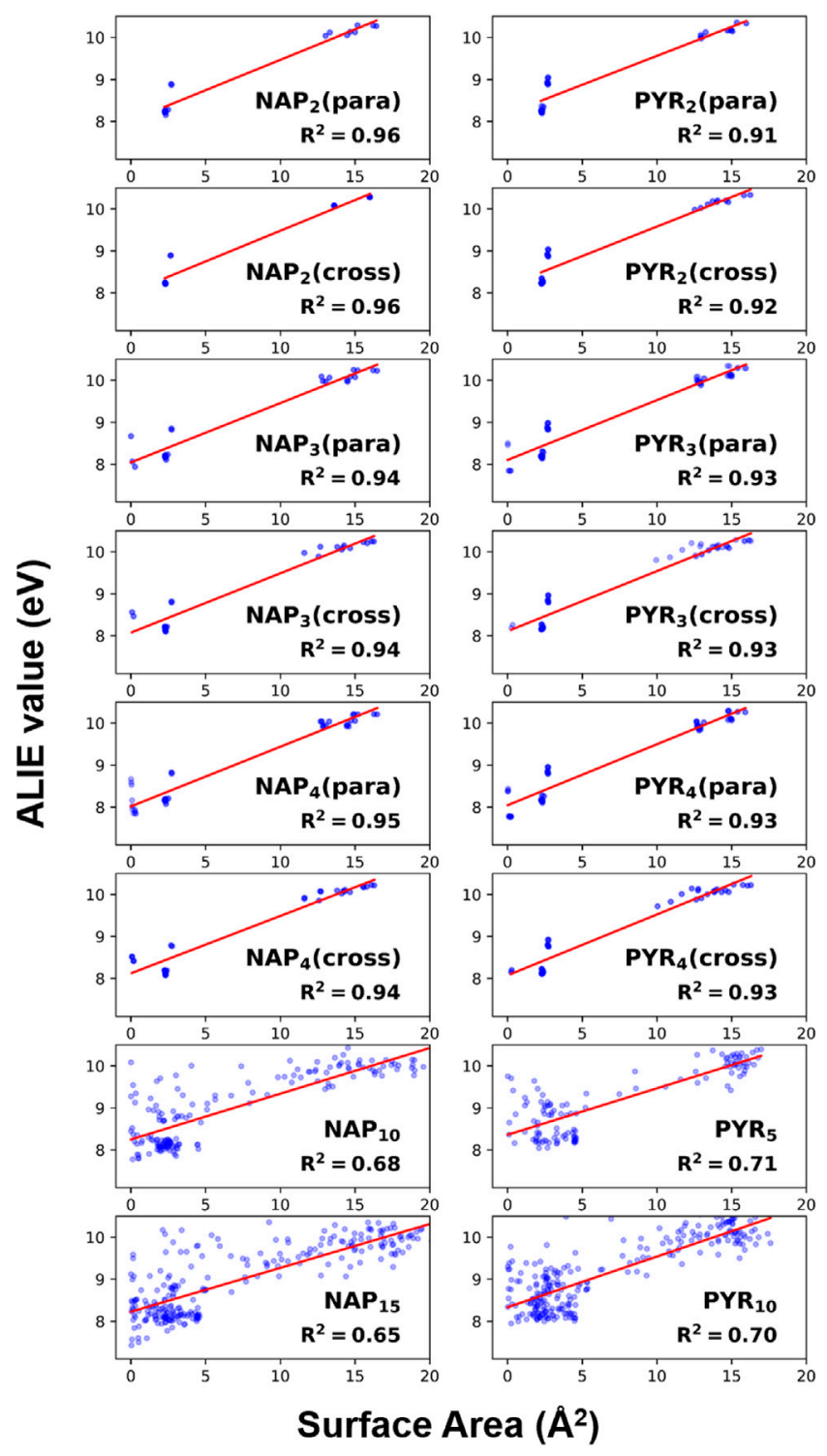

FIGURE 8 | The correlation of ALIE values with surface area of each atom. The red lines represent linear fittings, and the fitting quality is illustrated by $R^{2}$ shown in the plots.

clusters is computed at the B97D/6-311G(d,p) level of theory and the ALIE is extracted using Multiwfn (Lu and Chen, 2012). The numerical errors of reported ALIEs are within $0.03 \mathrm{eV}$, for example, some carbon atoms in NAP should share the same ALIE, but the optimized structure is not strictly symmetry causing an error of $0.03 \mathrm{eV}$ in the prediction of ALIE.

The location of the minimum ALIE is first discussed as it always refers to the most reactive sites. Figure 7 shows the spatial distribution of ALIE values within NAP and PYR clusters. The ALIE values are projected onto the iso-surface of electron density as $0.001 \AA^{-3}$. For the NAP monomer, the minimum ALIE locates on the top and bottom of the $p i$-plane, and the alpha site exhibits a slight higher reactivity, which is consistent with a previous work (Hou and You, 2017). Further increasing the $\mathrm{PAH}$ size to a tetramer, we see a minor decrease in the minimum ALIE. Also, it is noted that the parallel and crossed isomers share almost the same minimum ALIE, and the difference is within $0.03 \mathrm{eV}$. The minimum ALIE of NAP 10 and $\mathrm{NAP}_{15}$ clusters is 7.40 and $7.24 \mathrm{eV}$, respectively. According to a recent analysis of various aromatic species using ALIE (Martin et al., 2019), the most reactive sites in both $\mathrm{NAP}_{10}$ and $\mathrm{NAP}_{15}$ clusters shares similar chemical reactivity as localized $\pi$-radicals, which can cause potential crosslinking reactions. For PYR clusters, the changes in the minimum ALIE follow the same trend, and the value is reduced from 7.8 to $7.4 \mathrm{eV}$ considering a $\mathrm{PYR}_{10}$ cluster. In 
both cases, the minimum ALIE always locates at the surface pockets highlighting the enhanced reactivity within pockets. This is consistent with the indications from $\Delta E_{0}$ (Figure 6). Furthermore, it is interesting to note that the minimum ALIE of particles have a size dependence and a larger particle shows a lower minimum value. Therefore, it is evident that the surface reactivity of nascent soot is proportional to the particle size.

Figure 8 presents the correlation between ALIE values and surface area of each atom including both hydrogen and carbon atoms. The surface area is calculated from the iso-surface of electron density as $0.001 \AA^{-3}$ (Figure 7) (Bader et al., 1987). The ALIE values cluster into two groups in general (Figure 8); one group locates in the range of $0-5 \AA^{2}$, while the other group clusters around $15 \AA^{2}$. From the magnitude of surface area, we can classify the former group as the atoms in the surface pocket resulting the limited exposure. By contrast, the latter one refers to a group of bulk atoms, e.g., non-edge carbon in a PAH molecule. The atoms in the surface pocket show reduced ALIE values, indicating that the reactivity is higher than the bulk atoms. This finding supports our statement made from the analysis of reaction kinetics (Figure 6).

Besides, the reactivity of aromatics can be interpreted using HOMO-LUMO gap (Randić, 2003; Chen and Wang, 2019a). A lower HOMO-LUMO gap implies a higher reactivity. As we have known that HOMO-LUMO gaps of homogeneous clusters decrease with the growth in the particle size, which can be well captured by the quantum confinement effect (Liu C. et al., 2019; Chen and Wang, 2019b). As a result, the indications from HOMO-LUMO gaps further support our finding in both $\Delta E_{0}$ and ALIE that larger nascent soot particles have a higher surface reactivity.

Homogeneous clusters are used here as an example to mimic the nascent soot surface. It is well known that nascent soot has a complex molecular composition. For example, recent works reveal that $\mathrm{PAH}$ species in nascent soot carry functional groups including aliphatic and oxygenated groups using high resolution AFM (Commodo et al., 2019; Schulz et al., 2019). We expect that functional groups should have a minor impact on the surface reactivity owing to their weak interactions with radicals. Our finding here can be extended to explain the reactivity change in soot aging (Kholghy et al., 2016), where nascent soot has more irregular disordered surface pockets and thus its reactivity prevails. It is revealed that the most reactive sites locates in surface pockets, but the dynamics nature of pockets (Chen et al., 2015a) would impact reaction coordinates for surface reactions. However, this effect requires further investigations to establish a dynamic picture of surface reactions.

\section{CONCLUSION}

We compute the barrier height and reaction enthalpy of hydrogen abstraction reaction on small naphthalene, pyrene, coronene, ovalene and circumcoronene clusters by $\mathrm{H}$ radicals. Both clusters with a parallel and crossed configuration shows similar results as that of monomers, except naphthalene dimers with a crossed configuration having a reduced barrier height and reaction enthalpy. Moreover, it is shown that the barrier height is weakly proportional to the particle. Further calculations on larger PAH clusters (e.g., 1.1-1.9 nm) providing extra evidence to support the size dependence. In addition, surface sallow regions exhibit the lowest barrier height of hydrogen abstraction reaction, and the lowest value is $\sim 8.5 \mathrm{kcal} / \mathrm{mol}$, which is about the half of $\mathrm{PAH}$ monomers.

A set of model system is then generated to investigate the fundamental reason behind the reduction in barrier height for larger particles. It is found that the reduction in barrier height is attributed to the intermolecular interactions between neighboring atoms and the local curvature. Further ALIE analysis on $\mathrm{NAP}_{10}$ and $\mathrm{NAP}_{15}$ clusters supports the observations of size dependence in surface reactivity, where larger particles show a lower minimum ALIE value, indicating higher reactivity. Besides, it is shown that the minimum ALIE always locates in the surface pockets, in consistent with the results from barrier height. Therefore, it is concluded that the surface reactivity exhibits a size dependence, which is not previously noticed.

\section{DATA AVAILABILITY STATEMENT}

The original contributions presented in the study are included in the article/Supplementary Material, further inquiries can be directed to the corresponding author.

\section{AUTHOR CONTRIBUTIONS}

HW prepared the data in the manuscript and performed the detailed analysis, CY prepared several sets of data. DC wrote the manuscript and designed the project.

\section{FUNDING}

This paper is supported by the project of State Key Laboratory of Explosion Science and Technology (Beijing Institute of Technology). The project number is ZDKT21-0. DC also acknowledges the support by the National Natural Science Foundation of China (No. 21961122007 and 51806016).

\section{SUPPLEMENTARY MATERIAL}

The Supplementary Material for this article can be found online at: https://www.frontiersin.org/articles/10.3389/fmech.2021.738354/ full\#supplementary-material 


\section{REFERENCES}

Andersson, M. P., and Uvdal, P. (2005). New Scale Factors for Harmonic Vibrational Frequencies Using the B3LYP Density Functional Method with the Triple- $\zeta$ Basis Set 6-311+G (D, P). The J. Phys. Chem. A 109 (12), 2937-2941. doi:10.1021/jp045733a

Appel, J., Bockhorn, H., and Frenklach, M. (2000). Kinetic Modeling of Soot Formation with Detailed Chemistry and Physics: Laminar Premixed Flames of C2 Hydrocarbons. Combustion and Flame 121 (1), 122-136. doi:10.1016/ S0010-2180(99)00135-2

Bader, R. F. W., Carroll, M. T., Cheeseman, J. R., and Chang, C. (1987). Properties of Atoms in Molecules: Atomic Volumes. J. Am. Chem. Soc. 109 (26), 7968-7979. doi:10.1021/ja00260a006

Bowal, K., Martin, J. W., Misquitta, A. J., and Kraft, M. (2019b). Ion-Induced Soot Nucleation Using a New Potential for Curved Aromatics. Combustion Sci. Tech. 1, 1-19. doi:10.1080/00102202.2019.1565496

Bowal, K., Martin, J. W., and Kraft, M. (2019a). Partitioning of Polycyclic Aromatic Hydrocarbons in Heterogeneous Clusters. Carbon 143, 247-256. doi:10.1016/ j.carbon.2018.11.004

Camacho, J., Singh, A. V., Wang, W., Shan, R., Yapp, E. K. Y., Chen, D., et al. (2017). Soot Particle Size Distributions in Premixed Stretch-Stabilized Flat Ethylene-Oxygen-Argon Flames. Proc. Combustion Inst. 36 (1), 1001-1009. doi:10.1016/j.proci.2016.06.170

Camacho, J., Tao, Y., and Wang, H. (2015). Kinetics of Nascent Soot Oxidation by Molecular Oxygen in a Flow Reactor. Proc. Combustion Inst. 35 (2), 1887-1894. doi:10.1016/j.proci.2014.05.095

Chen, D., Akroyd, J., Mosbach, S., and Kraft, M. (2015a). Surface Reactivity of Polycyclic Aromatic Hydrocarbon Clusters. Proc. Combustion Inst. 35 (2), 1811-1818. doi:10.1016/j.proci.2014.06.140

Chen, D., Akroyd, J., Mosbach, S., Opalka, D., and Kraft, M. (2015b). Solid-liquid Transitions in Homogenous Ovalene, Hexabenzocoronene and Circumcoronene Clusters: A Molecular Dynamics Study. Combustion and Flame 162 (2), 486-495. doi:10.1016/j.combustflame.2014.07.025

Chen, D., and Luo, K. H. (2020). Reactive Sites on the Surface of Polycyclic Aromatic Hydrocarbon Clusters: A Numerical Study. Combustion and Flame 211, 362-373. doi:10.1016/j.combustflame.2019.09.034

Chen, D., Totton, T. S., Akroyd, J. W. J., Mosbach, S., and Kraft, M. (2014). Sizedependent Melting of Polycyclic Aromatic Hydrocarbon Nano-Clusters: A Molecular Dynamics Study. Carbon 67, 79-91. doi:10.1016/ j.carbon.2013.09.058

Chen, D., and Wang, H. (2019a). HOMO-LUMO Energy Splitting in Polycyclic Aromatic Hydrocarbons and Their Derivatives. Proc. Combustion Inst. 37 (1), 953-959. doi:10.1016/j.proci.2018.06.120

Chen, D., and Wang, H. (2019b). HOMO-LUMO Gaps of Homogeneous Polycyclic Aromatic Hydrocarbon Clusters. J. Phys. Chem. C 123 (45), 27785-27793. doi:10.1021/acs.jpcc.9b08300

Chen, S., Xiong, F., and Huang, W. (2019). Surface Chemistry and Catalysis of Oxide Model Catalysts from Single Crystals to Nanocrystals. Surf. Sci. Rep. 74 (4), 100471. doi:10.1016/j.surfrep.2019.100471

Cioslowski, J., Liu, G., Martinov, M., Piskorz, P., and Moncrieff, D. (1996). Energetics and Site Specificity of the Homolytic C-H Bond Cleavage in Benzenoid Hydrocarbons: An Ab Initio Electronic Structure Study. J. Am. Chem. Soc. 118 (22), 5261-5264. doi:10.1021/ja9600439

Commodo, M., Kaiser, K., De Falco, G., Minutolo, P., Schulz, F., D’Anna, A., et al. (2019). On the Early Stages of Soot Formation: Molecular Structure Elucidation by High-Resolution Atomic Force Microscopy. Combustion and Flame 205, 154-164. doi:10.1016/j.combustflame.2019.03.042

Dworkin, S. B., Zhang, Q., Thomson, M. J., Slavinskaya, N. A., and Riedel, U. (2011). Application of an Enhanced PAH Growth Model to Soot Formation in a Laminar Coflow Ethylene/air Diffusion Flame. Combustion and Flame 158 (9), 1682-1695. doi:10.1016/j.combustflame.2011.01.013

Frenklach, M. (1996). On Surface Growth Mechanism of Soot Particles. Symp. (International) Combustion 26 (2), 2285-2293. doi:10.1016/S0082-0784(96) 80056-7

Frenklach, M., and Wang, H. (1991). Detailed Modeling of Soot Particle Nucleation and Growth. Symp. (International) Combustion 23 (1), 1559-1566. doi:10.1016/ s0082-0784(06)80426-1
Frisch, M., Trucks, G., Schlegel, H., Scuseria, G., Robb, M., Cheeseman, J., et al. (2009). Gaussian 09, Revision D. 01. Wallingford CT: Gaussian, Inc.

Grančič, P., Martin, J. W., Chen, D., Mosbach, S., and Kraft, M. (2016). Can Nascent Soot Particles Burn from the inside? Carbon 109, 608-615. doi:10.1016/j.carbon.2016.08.025

Grimme, S., Antony, J., Ehrlich, S., and Krieg, H. (2010). A Consistent and Accurate $\mathrm{Ab}$ Initio Parametrization of Density Functional Dispersion Correction (DFT-D) for the 94 Elements H-Pu. J. Chem. Phys. 132 (15), 154104. doi:10.1063/1.3382344

Hou, D., and You, X. (2017). Reaction Kinetics of Hydrogen Abstraction from Polycyclic Aromatic Hydrocarbons by H Atoms. Phys. Chem. Chem. Phys. 19 (45), 30772-30780. doi:10.1039/C7CP04964A

Kholghy, M. R., Veshkini, A., and Thomson, M. J. (2016). The Core-Shell Internal Nanostructure of Soot - A Criterion to Model Soot Maturity. Carbon 100, 508-536. doi:10.1016/j.carbon.2016.01.022

Khosousi, A., and Dworkin, S. B. (2015). Soot Surface Reactivity during Surface Growth and Oxidation in Laminar Diffusion Flames. Combustion and Flame 162 (12), 4523-4532. doi:10.1016/j.combustflame.2015.09.005

Kopač, D., Likozar, B., and Huš, M. (2019). Catalysis of Material Surface Defects: Multiscale Modeling of Methanol Synthesis by $\mathrm{CO} 2$ Reduction on Copper. Appl. Surf. Sci. 497, 143783. doi:10.1016/j.apsusc.2019.143783

Lapuerta, M., Rodríguez-Fernández, J., Sánchez-Valdepeñas, J., and Science, C. (2020). Soot Reactivity Analysis and Implications on Diesel Filter Regeneration. Prog. Energ. Combustion Sci. 78, 100833. doi:10.1016/ j.pecs.2020.100833

Li, J., Lu, G., Wu, G., Mao, D., Guo, Y., Wang, Y., et al. (2014). Effect of TiO2 crystal Structure on the Catalytic Performance of $\mathrm{Co} 3 \mathrm{O} 4 / \mathrm{TiO} 2$ Catalyst for LowTemperature CO Oxidation. Catal. Sci. Technol. 4 (5), 1268-1275. doi:10.1039/ C3CY01004J

Liu, C., Singh, A. V., Saggese, C., Tang, Q., Chen, D., Wan, K., et al. (2019a). Flameformed Carbon Nanoparticles Exhibit Quantum Dot Behaviors. Proc. Natl. Acad. Sci. USA 116 (26), 12692-12697. doi:10.1073/pnas.1900205116

Liu, J.-X., Wang, P., Xu, W., and Hensen, E. J. M. (2017). Particle Size and Crystal Phase Effects in Fischer-Tropsch Catalysts. Engineering 3 (4), 467-476. doi:10.1016/J.ENG.2017.04.012

Liu, P., Li, Z., Bennett, A., Lin, H., Sarathy, S. M., and Roberts, W. L. (2019b). The Site Effect on PAHs Formation in HACA-Based Mass Growth Process. Combustion and Flame 199, 54-68. doi:10.1016/ j.combustflame.2018.10.010

Lu, T., and Chen, F. (2012). Multiwfn: A Multifunctional Wavefunction Analyzer. J. Comput. Chem. 33 (5), 580-592. doi:10.1002/jcc.22885

Macián, V., Serrano, J. R., Piqueras, P., and Sanchis, E. J. (2019). Internal Pore Diffusion and Adsorption Impact on the Soot Oxidation in wall-flow Particulate Filters. Energy 179, 407-421. doi:10.1016/j.energy.2019.04.200

Martin, J. W., Hou, D., Menon, A., Pascazio, L., Akroyd, J., You, X., et al. (2019). Reactivity of Polycyclic Aromatic Hydrocarbon Soot Precursors: Implications of Localized $\pi$-Radicals on Rim-Based Pentagonal Rings. J. Phys. Chem. C 123 (43), 26673-26682. doi:10.1021/acs.jpcc.9b07558

Pascazio, L., Martin, J. W., Botero, M. L., Sirignano, M., D’Anna, A., and Kraft, M. (2019). Mechanical Properties of Soot Particles: The Impact of Crosslinked Polycyclic Aromatic Hydrocarbons. Combustion Sci. Tech. 1, 1-21. doi:10.1080/ 00102202.2019 .1668380

Pfau, S. A., La Rocca, A., Fay, M. W., and Flame, M. (2020). Quantifying Soot Nanostructures: Importance of Image Processing Parameters for Lattice Fringe Analysis. Combustion and Flame 211, 430-444. doi:10.1016/ j.combustflame.2019.10.020

Randić, M. (2003). Aromaticity of Polycyclic Conjugated Hydrocarbons. Chem. Rev. 103 (9), 3449-3606. doi:10.1021/cr9903656

Schulz, F., Commodo, M., Kaiser, K., De Falco, G., Minutolo, P., Meyer, G., et al. (2019). Insights into Incipient Soot Formation by Atomic Force Microscopy. Proc. Combustion Inst. 37 (1), 885-892. doi:10.1016/j.proci.2018.06.100

Sediako, A. D., Soong, C., Howe, J. Y., Kholghy, M. R., and Thomson, M. J. (2017). Real-time Observation of Soot Aggregate Oxidation in an Environmental Transmission Electron Microscope. Proc. Combustion Inst. 36 (1), 841-851. doi:10.1016/j.proci.2016.07.048

Sharma, V., Uy, D., Gangopadhyay, A., O’Neill, A., Paxton, W. A., Sammut, A., et al. (2016). Structure and Chemistry of Crankcase and Exhaust Soot Extracted from Diesel Engines. Carbon 103, 327-338. doi:10.1016/j.carbon.2016.03.024 
Sjoberg, P., Murray, J. S., Brinck, T., and Politzer, P. (1990). Average Local Ionization Energies on the Molecular Surfaces of Aromatic Systems as Guides to Chemical Reactivity. Can. J. Chem. 68 (8), 1440-1443. doi:10.1139/v90-220

Totton, T. S., Misquitta, A. J., and Kraft, M. (2012). A Quantitative Study of the Clustering of Polycyclic Aromatic Hydrocarbons at High Temperatures. Phys. Chem. Chem. Phys. 14 (12), 4081-4094. doi:10.1039/C2CP23008A

Wan, K., Chen, D., and Wang, H. (2018). On Imaging Nascent Soot by Transmission Electron Microscopy. Combustion and Flame 198, 260-266. doi:10.1016/j.combustflame.2018.09.021

Wang, Y., Raj, A., and Chung, S. H. (2015). Soot Modeling of Counterflow Diffusion Flames of Ethylene-Based Binary Mixture Fuels. Combustion and Flame 162 (3), 586-596. doi:10.1016/j.combustflame.2014.08.016

Yu, H., and Truhlar, D. G. (2014). What Dominates the Error in the CaO Diatomic Bond Energy Predicted by Various Approximate Exchange-Correlation Functionals? J. Chem. Theor. Comput. 10 (6), 2291-2305. doi:10.1021/ ct5000814
Conflict of Interest: The authors declare that the research was conducted in the absence of any commercial or financial relationships that could be construed as a potential conflict of interest.

Publisher's Note: All claims expressed in this article are solely those of the authors and do not necessarily represent those of their affiliated organizations, or those of the publisher, the editors and the reviewers. Any product that may be evaluated in this article, or claim that may be made by its manufacturer, is not guaranteed or endorsed by the publisher.

Copyright $\odot 2021$ Wang, Chang and Chen. This is an open-access article distributed under the terms of the Creative Commons Attribution License (CC BY). The use, distribution or reproduction in other forums is permitted, provided the original author(s) and the copyright owner(s) are credited and that the original publication in this journal is cited, in accordance with accepted academic practice. No use, distribution or reproduction is permitted which does not comply with these terms. 